专家推介: 本文通过研究 AlGaN 日盲紫外像增强器的 MTF, 提出了紫外光在 AlGaN 光阴极内部传输时的散射、 光生载流子在 $\mathrm{AlGaN}$ 激活层中的散射以及发射电子的散射是影响 $\mathrm{AlGaN}$ 光阴极日盲紫外像增强管分辨力主要 因素的观点，对进一步提高 AlGaN 日盲紫外像增强器的分辨力具有一定的指导意义。

\title{
铝镓氮光阴极像增强器极限分辨力影响因素研究
}

间 否 1,2 , 石 峰 ${ }^{1,2}$, 单 聪 ${ }^{3}$, 程宏昌 ${ }^{1,2}$, 郭 欣 ${ }^{1,2}$, 刘 晖 ${ }^{1,2}$, 罗 洋 ${ }^{1,2}$, 张晓辉 1,2

(1. 微光夜视技术重点实验室, 陕西 西安 710065; 2. 昆明物理研究所, 云南 昆明 650223;

3. 陆军装备部驻西安地区军代局驻西安地区第八军事代表室, 陕西 西安 710065)

\begin{abstract}
摘要: 针对铝镓氮光阴极像增强器极限分辨力远小于同结构类型砷化镓光阴极像增强器极限分辨力的 问题, 基于紫外光激发荧光粉发光的特性, 搭建了铝镓氮光阴极的紫外光传输特性评测装置, 对铝镓 氮光阴极紫外光传输特性进行了测量, 并依据非衍射光学系统传函方程推算了铝镓氮光阴极的紫外光 学传递函数; 依据近贴聚焦系统调制传递函数方程, 并基于制备的铝镓氮光阴极像增强器的分辨力测 试数据, 推导了铝镓氮光阴极像增强器的前近贴聚焦系统调制传递函数方程; 通过对比研究铝镓氮光 阴极的紫外光调制传递函数方程和铝镓氮光阴极像增强管的前近贴聚焦系统调制传递函数方程对系 统传函影响的比例权重, 提出紫外光在铝镓氮光阴极内部传输时紫外光散射, 以及紫外光激发载流子 在铝镓氮激活层中的散射和发射电子散射均是造成铝镓氮光阴极像增强管极限分辨力低的因素, 且紫 外光激发载流子在铝镓氮激活层中的散射和发射电子散射是最主要的影响因素。
\end{abstract}

关键词: 极限分辨力; 铅镓氮光阴极; 像增强器

中图分类号：TN144 文献标识码：A 文章编号：1001-8891(2020)-08-0729-06

\section{Limiting Resolution of AIGaN Photocathode Image Intensifier Tube}

\author{
YAN Lei ${ }^{1,2}$, SHI Feng ${ }^{1,2}$, SHAN Cong ${ }^{3}$, CHENG Hongchang ${ }^{1,2}$, GUO Xin ${ }^{1,2}$, \\ LIU Hui ${ }^{1,2}$, LUO Yang ${ }^{1,2}$, ZHANG Xiaohui ${ }^{1,2}$ \\ (1. Science and Technology on Low-Light-Level Night Vision Laboratory, Xi'an 710065, China;
}

2. Kunming Institute of Physics, Kunming 650223, China;

3. No.8 Military Representative Office of PLA Army Armaments Department, Xi'an 710065, China)

\begin{abstract}
The limiting resolution of an AlGaN photocathode image intensifier tube is much less than the limiting resolution of a GaAs photocathode image intensifier tube; thus, based on the effect of ultraviolet fluorescence, we calculate the limit resolution of an AlGaN photocathode and promote its ultraviolet modulation transfer function. According to the proximity focus system modulation transfer function and the test result of the custom developed AlGaN photocathode image intensifier, the resolution model of the AlGaN photocathode image intensifier is established. By comparing the ultraviolet modulation transfer function with the proximity focus system modulation transfer function, we discovered that the ultraviolet and photoelectron transmission in an AlGaN photocathode can reduce the limiting resolution of the AlGaN photocathode image intensifier tube and that the main reason affecting the limiting resolution of the AlGaN photocathode image intensifier tube is the photoelectron transmission and the scatter in the AlGaN photocathode.
\end{abstract}

Key words: limiting resolution, AlGaN photocathode, image intensifier

\section{0 引言}

太阳光作为能量最强的自然光源，其直接或间接 产生的背景辐射对工作在近地表面的许多光电探测

收稿日期: 2019-12-19; 修订日期: 2020-01-17.

作者简介：间否（1986-)，男，高级工程师，硕士研究生，主要从事微光像增强器技术研究。E-mail：13572495775@163.com。 
设备都会造成严重干扰，但 200 320 nm 这段光谱区 太阳光辐射到达不了地面, 为所谓的 “日盲区”, 如 果让紫外探测系统工作在光谱区中的紫外波段, 就可 以使近地表面工作的紫外探测设备避开最强大的自 然光源, 在背景极其简单的条件下工作, 从而大大降 低信号处理的难度 ${ }^{[1-2]}$ 。

紫外预警利用 “目盲区” 来探测导弹的火焰与羽 烟, 在背景洁净的日盲区, 导弹羽烟的紫外辐射很容 易被检测出来。由于避开了最强的太阳辐射背景, 信 息处理负担明显减轻, 虚警率很低, 与红外预警相比, 不需要低温冷却, 体积小、重量轻、响应快 ${ }^{[1-2]}$ 。

铝镓氮光阴极可通过调节其发射层铝组分, 进而 使铝镓氮光阴极的禁带宽度可调, 实现铝镓氮光阴极 的日盲探测 ${ }^{[3-4]}$ 。铝镓氮光电阴极像增强器是目前最主 要的一种日盲型紫外探测器 ${ }^{[5-7]}$ 。

铝镓氮光阴极像增强器由铝镓氮光电阴极像增 强管和小型高压电源组成, 小型高压电源提供铝镓氮 光电阴极像增强管正常工作所需的电压, 而铝镓氮光 电阴极像增强管的优劣直接决定了铝镓氮光电阴极 像增强器的性能指标。极限分辨力指标是像增强管的 一项重要技术参数, 对于微光像增强管, 常用调制传 递函数 (modulation transfer function, MTF) 来评价 其分辨力特性。MTF 包含了对不同空间频率目标调制 度信息的传递能力。在光学像质评价中, 人们普遍认 为调制传递函数 MTF 是评价成像系统和器件像质的 最全面、最客观的方法, 而极限分辨力是器件 MTF 曲线 $3 \%$ 调制度对应的一个空间频率 ${ }^{[8]}$ 。铝镓氮光阴极 像增强管的结构与砷化镓光阴极像增强管的结构相 似, 但在相同工艺参数条件下, 铝镓氮光阴极像增强 管的极限分辨力远小于砷化镓光阴极的像增强管, 且 目前对铝镓氮光阴极紫外像增强器分辨力研究较少。 本文通过实验和理论结合的方法, 对紫外光在铝镓氮 光阴极中传输特性进行了测量, 并通过紫外光激发载 流子在铝镓氮激活层中的散射和发射电子散射特性 的理论分析, 推算确定了铝镓氮光阴极的光学调制传 递函数及其前近贴聚焦系统调制传递函数, 通过对比 这两个调制传递函数, 确定了导致铝镓氮光阴极像增 强管极限分辨力降低的主要因素。

\section{1 铝镓氮光阴极像增强器结构及其分辨力特性}

铝镓氮光阴极像增强器由铝镓氮光电阴极像增 强管和小型高压电源组成, 小型高压电源提供铝镓氮 光电阴极像增强管正常工作所需的电压, 而铝镓氮光 电阴极像增强管的优劣直接决定了铝镓氮光电阴极 像增强器的各项性能指标。
铝镓氮光阴极像增强管为近贴聚焦结构, 由光阴 极、微通道板 (MCP) 和荧光屏等部件组成, 结构如 图 1 所示, 其中光阴极面与 MCP 输入面之间称为第 一近贴距离 (first proximity distance), 用 $L_{1}$ 表示; $\mathrm{MCP}$ 输出面与荧光屏之间称为第二近贴距离 (second proximity distance), 用 $L_{2}$ 表示。

对于近贴聚焦结构的像增强管, 常采用调制传递 函数表示像增强管的分辨力特性, 像增强管的调制传 递函数可由式(1)表示:

$$
\begin{aligned}
\operatorname{MTF}_{\text {总 }}(f)= & \operatorname{MTF}_{\text {阴极 }}(f) \cdot \operatorname{MTF}_{1}(f) \cdot \operatorname{MTF}_{M C P}(f) \cdot \operatorname{MTF}_{2}(f) \cdot \\
& \operatorname{MTF}_{\text {屏 }(f)} \text { (1) }
\end{aligned}
$$

式中: $M_{T F}$ 阴极 $(f) 、 \operatorname{MTF}_{M C P}(f) 、 M T F$ 屏 $(f)$ 分别是光阴极、 微通道板、苂光屏调制传递函数; $\operatorname{MTF}_{1}(f) 、 \mathrm{MTF}_{2}(f)$ 分别是前、后近贴静电聚焦系统对应的调制传递函数; $f$ 代表空间频率，单位常为线对每毫米 $(\mathrm{lp} / \mathrm{mm})$ 。

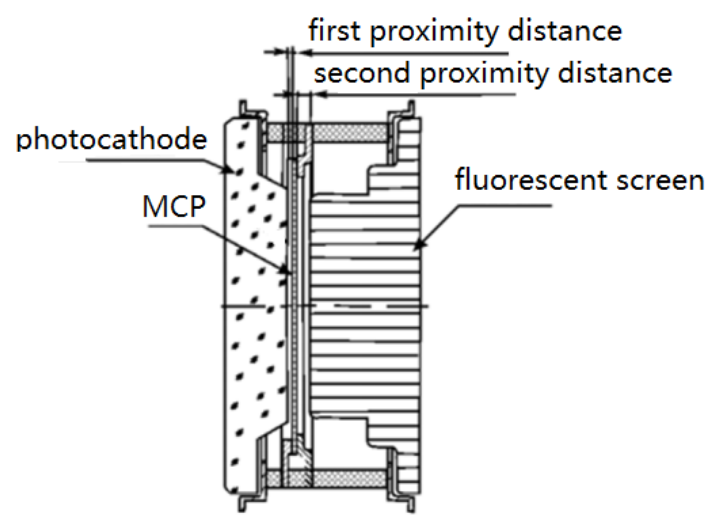

图 1 像增强管结构示意图

Fig.1 Structure diagram of image intensifier

关于近贴聚焦结构的像增强管分辨力研究方面, 目前较为成熟的是 GaAs 光阴极像增强管的分辨力特 性, 研究认为可见光和光激发载流子在 GaAs 光阴极 中的传输不是影响 GaAs 光阴极像增强管分辨力的主 要因素, MTF 阴极 $(f)$ 的影响可以忽略 ${ }^{[9-10]}$, 其调制传递 函数可由式(2)表示:

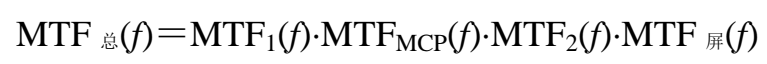

式中: $\operatorname{MTF}_{1}(f)$ 为前近贴聚焦系统调制传递函数, 表达 式为 $(3)^{[8]}$ :

$$
\mathrm{MTF}_{1}=\exp \left[-\frac{1}{11}\left(\pi f R_{\mathrm{mp}}\right)^{2}\right]
$$

式中: $R_{\mathrm{mp}}=2 L_{1} \sqrt{\frac{\varepsilon_{\mathrm{m}}}{\phi}} ; \varepsilon_{\mathrm{m}}$ 为光阴极发射光电子最大初 始能量, $\varepsilon_{\mathrm{m}}=h c\left(\frac{1}{\lambda_{1}}-\frac{1}{\lambda_{2}}\right)=1.73 \mathrm{eV} ; \phi_{1}$ 为光阴极与微 通道板输入面之间的电压值。

$\mathrm{MTF}_{\mathrm{MCP}}(f)$ 为 MCP 的调制传递函数, 表达式为 $(4)^{[8]}$ :

$$
\operatorname{MTF}_{\mathrm{MCP}}=J_{1}(2 \pi f d) / \pi f d
$$


式中: $d$ 为 MCP 的丝芯距尺寸。

$\mathrm{MTF}_{2}(f)$ 为后近贴聚焦系统的调制传递函数, 表达 式为 $(5)^{[8]}$ :

$$
\mathrm{MTF}_{2}=\exp \left[-\frac{1}{9} \pi^{2} f^{2} L_{2}^{2} \frac{\varepsilon_{\mathrm{m}_{2}}}{\phi_{2}}\right]
$$

式中: $\varepsilon_{\mathrm{m}_{2}}$ 为 MCP 发射电子最大初始能量, 一般认为 该值为 $5 \mathrm{eV} ; \phi_{2}$ 为 $\mathrm{MCP}$ 输出面与荧光屏之间的电压值。

$\mathrm{MTF}$ 屏 $(f)$ 为荧光屏的调制传递函数, 表达式为 $(6)^{[8]}:$

$$
\mathrm{MTF}_{\text {屏 }}=\exp \left[-\left(\frac{f}{46}\right)^{1.1}\right]
$$

铝镓氮光阴极光电子发射机制与砷化镓光阴极 相同 ${ }^{[6]}$, 且砷化镓光阴极与铝镓氮光阴极结构相似, 图 2、图 3 为两种像增强管光阴极的结构示意图。

鉴于这两种像增强管结构的相似性, 前期在对铝 镓氮光阴极紫外像增强管分辨力研究时, 均借鉴砷化 镓光阴极像增强管的调制传递函数方程。按照砷化镓 光阴极像增强管的调制传递函数对铝镓氮光阴极像 增强管的极限分辨力进行计算, 可得铝镓氮光阴极像 管在特定参数条件下其理论极限分辨力如表 1 所示。

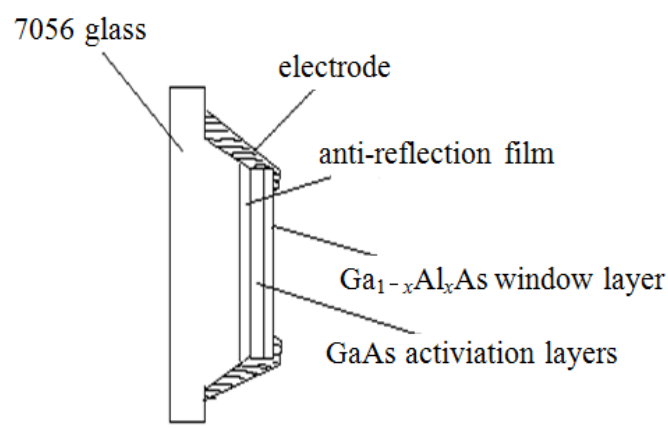

图 2 微光像管光阴极结构示意图

Fig.2 Structure diagram of GaAs photocathode
因在实际工艺中, 两种像增强管的差异仅为光阴 极, 其几何参数中除了 $L_{1}$ 存在差异外, 其余几何参数 均一致, 因此表 1 中仅给出了 $L_{1}$ 有差异时的情况。

但实际制备的 $\mathrm{AlGaN}$ 光阴极像增强管, 即使其第 一近贴距离参数 $L_{1}$ 达到 $0.13 \mathrm{~mm}$, 像管的其他参数按 照表 1 所述设定，制备像管的分辨力远低于 $61 \mathrm{lp} / \mathrm{mm}$ 的计算值。表 2 为项目研制期间制备的部分像管分辨 力测试值。

通过对比表 1 中按照砷化镓光阴极像管分辨力模 型计算铝镓氮光阴极像管得到的理论分辨力值与表 2 中铝镓氮光阴极像管实测分辨力值的数据可知, 分辨 力理论计算值近乎为实测值的 2 倍, GaAs 光阴极微 光像增强管的调制传递函数方程无法直接在 $\mathrm{AlGaN}$ 光阴极像增强管中应用。由于这两种像增强管的差异 仅为光阴极材料的差异, 因此对于 $\mathrm{AlGaN}$ 光阴极像 增强管分辨力特性研究时, 可借鉴 GaAs 光阴极微光 像增强管关于 $\operatorname{MTF}_{\mathrm{MCP}}(f) 、 \mathrm{MTF}_{2}(f) 、 \mathrm{MTF}$ 屏 $(f)$ 这 3 项 数学方程, 需要对 $\mathrm{MTF}$ 阴极 $(f) 、 \mathrm{MTF}_{1}(f)$ 这两项数学方 程重新确定, 即紫外光在 $\mathrm{AlGaN}$ 光阴极内部传输的 散射特性，及紫外光激发载流子在 $\mathrm{AlGaN}$ 激活层的 散射和发射电子散射需要重新进行研究。

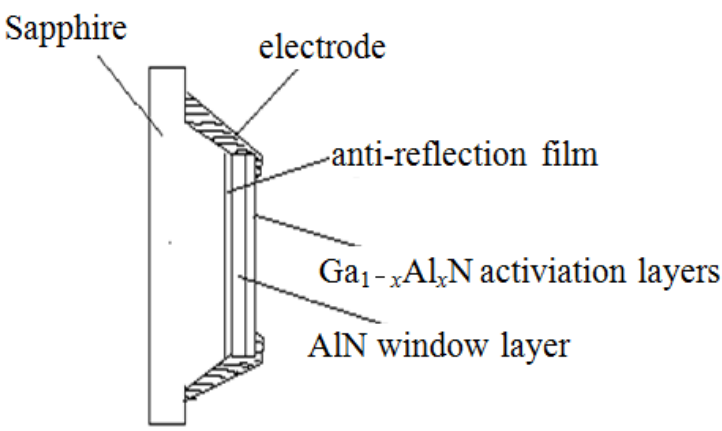

图 3 紫外像管光电阴极结构示意图

Fig.3 Structure diagram of AlGaN photocathode 表 1 不同参数时铝镓氮光阴极像管极限分辨力理论值

Table 1 Theoretical value of limit resolution of AlGaN photocathode with different parameters

\begin{tabular}{ccccccc}
\hline Image intensifier & $L_{1} / \mathrm{mm}$ & $\phi_{1} / \mathrm{V}$ & $d / \mu \mathrm{m}$ & $L_{2} / \mathrm{mm}$ & $\phi_{2} / \mathrm{kV}$ & Limit resolution $/(\mathrm{lp} / \mathrm{mm})$ \\
\hline $1 \#$ & 0.15 & 400 & 8 & 0.5 & 5 & 51 \\
$2 \#$ & 0.14 & 400 & 8 & 0.5 & 5 & 54 \\
$3 \#$ & 0.13 & 400 & 8 & 0.5 & 5 & 61 \\
\hline
\end{tabular}

表 2 铝镓氮光阴极像管分辨力测试情况

Table 2 Measured resolution of AlGaN photocathode image tube

\begin{tabular}{cccc}
\hline No. & $L_{1} / \mathrm{mm}$ & $L_{2} / \mathrm{mm}$ & Limit resolution $/(\mathrm{lp} / \mathrm{mm})$ \\
\hline $1 \#$ & 0.13 & 0.5 & 34.6 \\
$2 \#$ & 0.13 & 0.5 & 34.6 \\
$3 \#$ & 0.12 & 0.5 & 38.6 \\
\hline
\end{tabular}




\section{2 紫外光在铝镓氮光阴极内部散射特性研究}

鉴于目前尚无紫外光学调制传递函数测量设备, 因此无法直接通过测传函的方法对铝镓氮光阴极的 传函特性进行评价。针对该项困难, 搭建了一套铝镓 氮光阴极紫外分辨力特性测试的装置, 测量装置原理 图如图 4 所示。

测试过程如下:

1) 将涂单层苂光粉的光纤面板放置 (以下简称 “涂粉面板”) 在图 4 所示涂粉面板的位置, 通过调节 装置调节被测件与涂粉面板间距，控制在 $0.1 \mathrm{~mm} \pm$ $0.02 \mathrm{~mm}$ 范围;

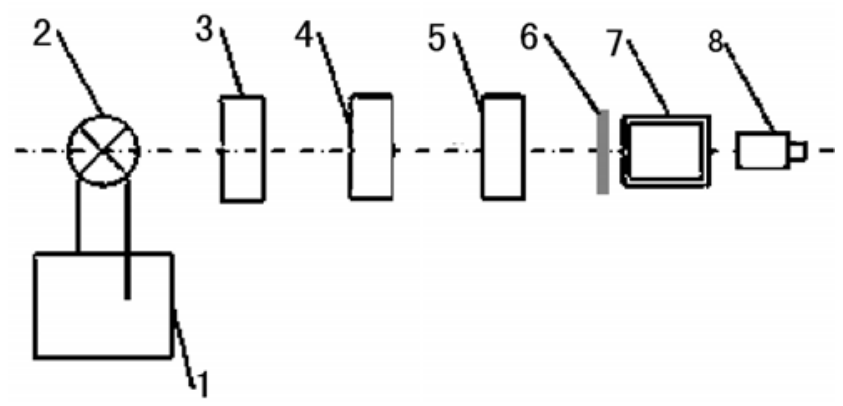

图 $4 \mathrm{AlGaN}$ 光阴极紫外分辨力特性测试的装置示意图

Fig.4 Schematic diagram of AlGaN photocathode UV resolution characteristic test device

注: 1.気灯光源 2.気灯 3. 紫外带通滤光片 4. 紫外分辨力板 5.投影光学系统 6.被测件 7.涂单层苂光粉的光纤面板 8.观察系统

Note: 1. Deuterium lamp house; 2. Deuterium lamp; 3. Ultraviolet band pass filter; 4. UV resolution target plate; 5 . Projection optical system; 6. Test piece; 7. Fiber optic panel coated with single layer phosphor; 8.Observation system

2) 开启気灯光源, 使紫外光透过被测件并在涂 粉面板上成像;

3）调节观察系统, 读取观察系统可分辨的最小 可辨识分划板刻线，并记录;

4）去除被测件, 使得紫外光直接激发涂粉靶板, 调节观察系统, 读取观察系统可分辨的最小可辨识分 划板刻线，并记录。

实验共对 5 片 $\mathrm{AlGaN}$ 光阴极的分辨力特性进行 测量, 测试结果如表 3 所示。

而在去除 $\mathrm{AlGaN}$ 光阴极后, 仅对涂粉面板的极 限分辨力进行测试时, 读取的极限分辨力值为 112 $\mathrm{lp} / \mathrm{mm}$ 。

根据测试结果判断铝镓氮光阴极自身对紫外光 有较大的散射。根据文献[11], 苂光屏调制传递函数 满足公式(6)。
表 3 铝镓氮光阴极的极限分辨力读数

Table 3 Limit resolution of AlGaN photocathode

\begin{tabular}{ccc}
\hline No. & Photocathode & Limit resolution \\
\hline 1 & $1 \#$ & 67 \\
2 & $2 \#$ & 64 \\
3 & $3 \#$ & 61 \\
4 & $4 \#$ & 67 \\
5 & $5 \#$ & 67 \\
\hline
\end{tabular}

按照式(6)计算得分辨力为 $67 \mathrm{lp} / \mathrm{mm}$ 时荧光屏的 传函值为 $22 \%$, 又根据调制传递函数为 $3 \%$ 时为人眼 的极限分辨力, 推算出 $\mathrm{AlGaN}$ 光阴极在 $67 \mathrm{lp} / \mathrm{mm}$ 时 的传函值为 $9 \%$ 。根据非衍射限光学系统的调整传递 函数 ${ }^{[6]}$, 可得到 $\mathrm{AlGaN}$ 光阴极的光学调制传递函数方 程, 即 $M T F$ 阴极满足式(7):

$$
\operatorname{MTF}_{\text {阴极 }}=\exp \left[-2 \pi^{2} \delta^{2} f^{2}\right]
$$

式中: $\delta$ 为材料特性参数, 为 $5.2 \times 10^{-3} ; f$ 为频率参数。

\section{3 铝镓氮紫外像增强管前近贴聚焦系统的分 辨力特性分析}

根据近贴聚焦系统 MTF 的函数表达式 ${ }^{[10]}$ ，像增 强管前近贴聚焦系统的 MTF 满足式(8):

$$
\mathrm{MTF}_{1}=\exp \left[-\omega\left(\pi f R_{\mathrm{mp}}\right)^{2}\right]
$$

式中: $\omega$ 是与光阴极中载流子散射及最终发射电子散 射特性相关的参数。

根据半导体中载流子输运时的平均自由程, 半 导体内部激发光电子每碰撞一次的平均距离约为 2 10 nm, 单次碰撞能量损失约为 $0.01 \mathrm{eV}$ 。对于 $\mathrm{GaAs}$ 光阴极, 其激活层厚度在 $1.5 \mu \mathrm{m}$ 左右, 因此在垂直于 光阴极激活面垂直方向, 光电子从激活到发射约经历 150 次碰撞, 光电子能量损失约为 $1.5 \mathrm{eV}$, 而对于沿 其他方向输运的光电子, 碰撞次数更多, 能量损失更 大, 因此发射几率减小, 因此对于 GaAs 光阴极, 其 光电子发射主要沿光阴极激活面垂直方向, 根据经 验, GaAs 光阴极光电子发射可由 Lambert 和 Beta 模 型较好地描述, $\mathrm{GaAs}$ 光阴极前近贴静电聚焦系统对 应的传递函数表达式，如式(9) ${ }^{[10]}$ 所示:

$$
\mathrm{MTF}_{1}=\exp \left[-\frac{1}{11}\left(\pi f R_{\mathrm{mp}}\right)^{2}\right]
$$

对于 $\mathrm{AlGaN}$ 光阴极, 其激活层厚度仅为 $50 \mathrm{~nm}$, 光阴极体内受光子激发到导带中的过热电子在向真 空界面传输的过程中碰撞次数远小于 GaAs 光阴极, 故其发射光电子的能量更大, 另外, 由于 AlGaN 激 
活层材料生长缺陷较多 ${ }^{[12]}$, 载流子在输运过程中散射 的概率增加, 因此达到激活层表面的光电子发射角更 加离散, 公式(9)不再适用于 $\mathrm{AlGaN}$ 光阴极, 需要对 公式(8)中的 $\omega$ 参数重新进行求解。

将公式(4)、(5)、(6)、(7)、(8)代入公式(1), 可得 $\mathrm{AlGaN}$ 光阴极像增强管的调制传递函数公式(10):

$$
\begin{aligned}
\mathrm{MTF}_{\text {总 }}= & \exp \left[-2(\pi \sigma f)^{2}\right] \exp \left[-\omega\left(\pi f R_{\mathrm{mp}}\right)^{2}\right] \times \\
& J_{1}(2 \pi f d) / \pi f d \times \exp \left[-\frac{1}{9} \pi^{2} f^{2} L_{2}^{2} \frac{\varepsilon_{\mathrm{m} 2}}{\phi_{2}}\right] \exp \left[-\left(\frac{f}{4.6}\right)^{1.1}\right]
\end{aligned}
$$

为获得 $\mathrm{AlGaN}$ 光阴极像增强管对应的前近贴聚 焦系统的调制传递函数表达式, 我们利用极限分辨力 与调制传递函数的对应关系，根据制备 $\mathrm{AlGaN}$ 光阴 极像增强管的实测极限分辨力值推算出 $\omega$ 参数, 并对 $\omega$ 求平均值, 求得参数 $\omega$ 为 0.8 。为说明该方法计算参数 $\omega$ 的准确性, 将该参数带入方程(10), 计算制备 AlGaN 光阴极像增强管的理论分辨力, 并将该值与其实测值 比对，如表 4 所示。

表 4 制备的铝镓氮光阴极像增强管极限分辨力对比表

Table 4 Comparison table of limit resolution of AlGaN photocathode image intensifier tube

\begin{tabular}{cccc}
\hline & \multirow{2}{*}{\begin{tabular}{c} 
Image \\
\cline { 3 - 4 } No.
\end{tabular}} & \multicolumn{2}{c}{ Limit resolution/(lp/mm) } \\
\cline { 3 - 4 } & intensifier & $\begin{array}{c}\text { measured } \\
\text { value }\end{array}$ & $\begin{array}{c}\text { theoretical } \\
\text { value }\end{array}$ \\
\hline 1 & $1 \#$ & 38.6 & 41 \\
2 & $2 \#$ & 34.6 & 41 \\
3 & $3 \#$ & 34.6 & 40 \\
4 & $4 \#$ & 38.6 & 38 \\
5 & $5 \#$ & 38.6 & 39 \\
6 & $6 \#$ & 34.6 & 38 \\
\hline
\end{tabular}

根据表 4 , 确定的参数 $\omega$ 可较好地拟合铝镓氮光 阴极像增强管的极限分辨力实测值, 因此铝镓氮光阴 极像增强管前近贴聚焦系统的传递函数可表示为下 式:

$$
\begin{gathered}
\mathrm{MTF}_{1}=\exp \left[-0.8\left(\pi f R_{\mathrm{mp}}\right)^{2}\right] \\
R_{\mathrm{mp}}=2 \mathrm{~L} \sqrt{\frac{\varepsilon_{\mathrm{m}}}{\phi}}
\end{gathered}
$$

式中: $\varepsilon_{\mathrm{m}}$ 为 $2.07 \mathrm{eV}, \varepsilon_{\mathrm{m}}=h c\left(\frac{1}{\lambda_{1}}-\frac{1}{\lambda_{2}}\right) ; \lambda_{1}$ 和 $\lambda_{1}$ 为铝 镓氮像增强管响应起始和截止波长。

\section{4 铝镓氮光阴极像增强管极限分辨力影响因 素分析}

利用公式(11)替代公式(10)中的对应项, 可以求得 $\mathrm{AlGaN}$ 光阴极像增强管完整的调制传递函数表达式, 如公式(13):

$$
\begin{aligned}
\operatorname{MTF}_{\text {总 }} & \exp \left[-2(\pi \sigma f)^{2}\right] \exp \left[-0.8\left(\pi f R_{\mathrm{mp}}\right)^{2}\right] \times \\
& J_{1}(2 \pi f d) / \pi f d \times \exp \left[-\frac{1}{9} \pi^{2} f^{2} L_{2}^{2} \frac{\varepsilon_{\mathrm{m} 2}}{\phi_{2}}\right] \exp \left[-\left(\frac{f}{4.6}\right)^{1.1}\right]
\end{aligned}
$$

设定如下参数, $L_{1}=0.13 \mathrm{~mm}, \phi_{1}=400 \mathrm{~V}, d=6 \mu \mathrm{m}$, $L_{2}=0.5 \mathrm{~mm}, \phi_{2}=5000 \mathrm{~V}$, 并将这些参数分别代入公 式(2)和公式(13), 可得 $\mathrm{AlGaN}$ 光阴极像增强管和 GaAs 光阴极像增强管的调制传递函数曲线，如图 5 所示。

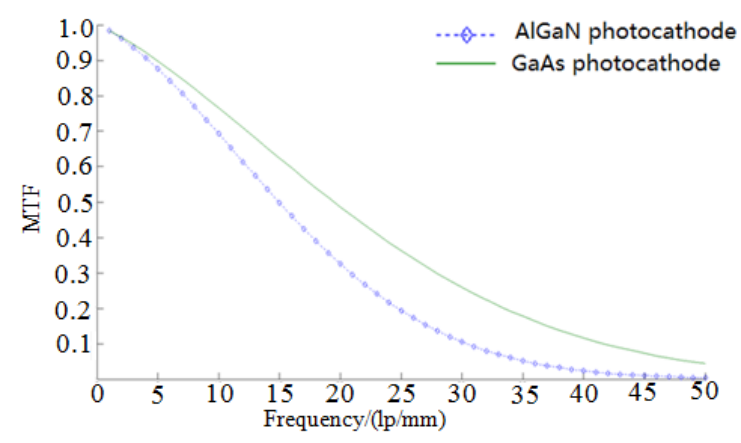

图 5 两种像增强管调制传递函数曲线对比

Fig.5 Comparison of modulation transfer function curves of two image intensifiers

根据图 5, 在相同条件参数时, $\mathrm{AlGaN}$ 光阴极像 增强管的调制传递函数值明显小于 GaAs 光阴极像增 强管的调制传递函数值，根据上述章节的实验和理论 分析, 引起 $\mathrm{AlGaN}$ 光阴极像增强管的调制传递函数 值偏低的原因为紫外光在 $\mathrm{AlGaN}$ 光阴极内部传输的 散射, 及紫外光激发载流子在 $\mathrm{AlGaN}$ 激活层的散射 和发射电子散射。为了确定这两项因素的权重, 特根 据表达式(8)、(11), 计算相同空间频率时其各自对应 的调制传递函数值，计算结果如表 5 所示。

根据表 5 , 随着频率增加, $\mathrm{MTF}_{1}$ 的影响作用变大, 当空间频率为 $40 \mathrm{lp} / \mathrm{mm}$ 时, $\mathrm{MTF}_{1}$ 的数值不足 $\mathrm{MTF}$ 阴极 的一半, 因此可以推断紫外光激发载流子在 $\mathrm{AlGaN}$ 激活层的散射和发射电子散射是引起 AlGaN 光阴极 像增强管极限分辨力低的主要原因。

\section{5 结论}

本文基于搭建的铝镓氮光阴极的紫外光传输特 性测量装置, 以及本单位制备的铝镓氮光阴极像增强 
表 5 调制传递函数值对比表

Table 5 Correlation table of MTF

\begin{tabular}{cccc}
\hline No. & Frequency/(lp/mm) & $\mathrm{MTF}_{\text {photocathode }}$ & $\mathrm{MTF}_{1}$ \\
\hline 1 & 0 & 1 & 1 \\
2 & 5 & $98.9 \%$ & $97.7 \%$ \\
3 & 10 & $95.7 \%$ & $91.2 \%$ \\
4 & 15 & $90.7 \%$ & $81.3 \%$ \\
5 & 20 & $84.0 \%$ & $69.2 \%$ \\
6 & 25 & $76.2 \%$ & $56.3 \%$ \\
7 & 30 & $67.6 \%$ & $43.7 \%$ \\
8 & 35 & $58.7 \%$ & $32.4 \%$ \\
9 & 40 & $49.8 \%$ & $23.0 \%$ \\
\hline
\end{tabular}

管的分辨力测试数据, 推算确定了铝镓氮光阴极像增 强管的调制传递函数方程, 并通过对比铝镓氮光阴极 的紫外光调制传递函数方程和铝镓氮光阴极像增强 管的前近贴聚焦系统调制传递函数方程, 确定随着频 率增加, 紫外光激发载流子在铝镓氮激活层的散射和 发射电子散射对铝镓氮光阴极像增强管极限分辨力 影响作用越大, 当空间频率为 $40 \mathrm{lp} / \mathrm{mm}$ 时, 其调制传 递函数值仅为 $23 \%$, 因此, 紫外光激发载流子在铝镓 氮激活层的散射和发射电子散射是造成铝镓氮光阴 极像增强管极限分低的主要原因。

\section{参考文献:}

[1] 许强. 军用紫外探测技术及应用[M]. 北京: 北京航空航天大学出版社, 2010.

XU Qiang. Military Ultraviolet Detection Technology and Its Application[M]. Beijing: Beijing University of Aeronautics and Astronautics Press, 2010.

[2] 石峰, 程宏昌, 闵否. 紫外探测器技术[M]. 北京: 国防工业出版社, 2017.

SHI Feng, CHENG Hongchang, YAN Lei. UV Detection Technique[M]. Beijing: National Defence Industrial Press, 2017.

[3] 付小倩, 常本康, 李䒨, 等. 负电子亲和势 $\mathrm{GaN}$ 光电阴极的研究进展 [J]. 物理学报, 2011, 60(3): 038503.

FU Xiaoqing, CHANG Benkang, LI Biao, et al. Progress of negative electron affinity GaN photocathode[J]. Acta Phys. Sin., 2011, 60(3): 038503.
[4] 李杀, 常本康, 徐源, 等. GaN 光电阴极的研究及其发展 [J]. 物理学报, 2011, 60(8): 088503.

LI Biao, CHANG Benkang, XU Yuan, et al. Research and development of GaN photocathode[J]. Acta Phys. Sin., 2011, 60(8): $088503 .$.

[5] 李慧芯, 申屠军, 戴丽英, 等. 负电子亲和势氮化镓光电阴极 [J]. 光电 子技术, 2007, 27(2): 73-77

LI Huirui, SHEN Tujun, DAI Liying, et al. GaN based negative electron affinity photocathode[J]. Optoelectronic Technology, 2007, 27(2): 73-77.

[6] 乔建良, 牛军, 杨智, 等. NEA GaN 光电阴极表面模型研究 [J]. 光学 技术, 2009, 35(1): 145-147.

QIAO Jianliang, NIU Jun, YANG Zhi, et al. Study of NEA GaN photocathode surface model[J]. Optical Technique, 2009, 35(1):145-147.

[7] 曾正清, 李朝木, 王宝林, 等. GaN 负电子亲和势光电阴极的激活改进 研究[J]. 真空与低温, 2010, 16(2): 108-112.

ZENG Zhengqing, LI Chaomu, WANG Baolin, et al. Investigation improvement activation of $\mathrm{GaN}$ negative electron affinity photocathodes[J]. Vacuum and Cryogenics, 2010, 16(2): 108-112.

[8] 程耀进, 向世明, 师宏立. 三代微光像增强器分辨力计算理论模型[J]. 应用光学, 2007, 28(5): 578-581

CHENG Yaojin, XIANG Shimin, SHI Hongli. Theoretical model for resolution calculation of third generation image intensifiers[J]. Journal of Applied Optics, 2007, 28(5): 578-581.

[9] 任玲. GaAs 光电阴极及像增强器的分辨力研究[D]. 南京: 南京理工大 学, 2013 .

REN Ling. Research on the Resolution of GaAs Photocathode and Image Intensifier[D]. Nanjing: Nanjing University of Science \& Technology, 2013.

[10] 向世明, 倪国强. 光电子成像器件原理[M]. 北京: 国防工业出版社, 1999.

XIANG Shiming, NI Guoqiang. Principle of Optoelectronic Imaging Device[M]. Beijing: National Defence Industrial Press, 1999.

[11] 邹异松, 刘玉凤, 白廷柱. 光电成像原理[M]. 北京: 北京理工大学出 版社, 1997.

ZOU Yisong, LIU Yufeng, BAI Tingzhu. Principle of Photoelectric Imaging[M]. Beijing: Beijing Institute of Technology Press, 1997.

[12] 李朝木, 曾正清, 陈群霞, 等. GaN 负电子亲和势光电阴极材料的生 长研究[J]. 真空与低温, 2008, 14(4): 236-239.

LI Chaomu, ZENG Zhengqing, CHEN Qunxia, et al. Investigation growth of GaN negative electron affinity photocathodes material[J]. Vacuum and Cryogenics, 2008, 14(4): 236-239. 